Dear editor and referees.

I would like to thank your effort. Thanks to the time you dedicate to highlight the weak points of this work the final document is much better than when it was firstly submitted.

We have tracked and corrected the errors mentioned by referee \#2. You will be able to check that in the attached files.

Best regards on behalf of all the authors

Dr Víctor Vega-Mayoral 


\title{
Charge trapping and coalescence dynamics in few layer $\mathrm{MoS}_{2}$
}

\author{
Victor Vega-Mayoral, ${ }^{* a, b, c}$ Tetiana Borzda, ${ }^{a, b}$ Daniele Vella, ${ }^{a, b, d}$ Matej Prijatelj, ${ }^{a, b}$ \\ Eva A. A. Pogna, ${ }^{e}$ Claudia Backes,,${ }^{c, f, g}$ Jonathan N Coleman, ${ }^{c, f}$ Giulio Cerullo, ${ }^{e}$ Dragan Mihailovic ${ }^{a, b, h}$ \\ and Christoph Gadermaier ${ }^{a, b, *}$ \\ *E-mail:vegamayv@tcd.ie \\ E-mail: christoph.gadermaier@ijs.si \\ ${ }^{a}$ Department of Complex Mater, Jozef Stefan Institute, Jamova cesta 39, 1000, Ljubljana, Slovenia \\ ${ }^{b}$ Jozef Stefan International Postgraduated School, Jamova cesta 39, 1000, Ljubljana, Slovenia \\ ${ }^{c}$ School of Physics, Trinity College Dublin Dublin 2, Ireland \\ ${ }^{d}$ Department of Physics, National University of Singapore, 2 Science Drive 3, Singapore 117542, Singapore \\ ${ }^{e}$ IFN-CNR, Department of Physics, Politecnico di Milano, P. Leonardo da Vinci 32, 20133 Milan, Italy \\ ${ }^{f}$ CRANN \& AMBER, Trinity College Dublin Dublin 2, Ireland \\ ${ }^{g}$ Applied Physical Chemistry department, Im Neuenheimer Feld 253, 69120 Heidelberg, Germany \\ ${ }^{h}$ Center of Excellence in Nanoscience and Nanotechnology, Jamova 39, 1000 Ljubljana, Slovenia
}

The optoelectronic properties of a material are determined by the processes following light-matter interaction. Here we use femtosecond optical spectroscopy to systematically study photoexcited carrier relaxation in few-layer $\mathrm{MoS}_{2}$ flakes as a function of excitation density and sample thickness. We find bimolecular coalescence of charges into indirect excitons as the dominant relaxation process in two- to three-layer flakes while thicker flakes show a much higher density of defects, which efficiently trap charges before they can coalesce.

\section{Introduction}

Transition metal dichalcogenides (TMDCs) are two-dimensional layered semiconductors consisting of a hexagonally packed layer of a transition metal M (typically Mo or W) sandwiched between two layers of chalcogen atoms $\mathrm{X}$ (typically $\mathrm{S}$ or Se) and interacting through strong covalent bonds, forming a $\mathrm{MX}_{2}$ compound. In multilayer TMDCs, the single layers are loosely bound to each other by van-der-Waals interactions. The best studied member of the family is the earth abundant $\mathrm{MoS}_{2}$, a semiconductor whose gap ranges from $1.3 \mathrm{eV}$ indirect in the bulk to $1.9 \mathrm{eV}$ direct in the monolayer ${ }^{1,2}$. The demonstration of high mobility monolayer transistors ${ }^{3}$ and the facile processing using liquid phase exfoliation ${ }^{4}$ (LPE) triggered vigorous research activity on semiconducting TMDCs ${ }^{5-11}$. Their strong light-matter interaction makes them particularly interesting also for photonic applications such as saturable absorbers ${ }^{12}$, electromodulators ${ }^{13}$ second harmonic generators ${ }^{14}$, and light emitting devices ${ }^{15}$.

The photonic properties of an active material originate in the behaviour of photoexcited states, which typically manifests itself on the fs to ps time scales. Femtosecond optical pump-probe spectroscopy has been extensively employed to gain insight into the photoexcitation dynamics in semiconducting TMDCs, such as exciton dissociation in few-layer $\mathrm{MoS}_{2}{ }^{16}$, carrier thermalization and cooling in few-layer $\mathrm{MoS}_{2}{ }^{17,18}$, and bimolecular exciton annihilation in monolayer $\mathrm{MoS}_{2}{ }^{19}$. Each of these processes has been studied on a different type of sample under different excitation conditions, which has so far precluded the merging of these patches of insight into a coherent picture.

Here we use broadband femtosecond pump-probe spectroscopy to study the whole cascade from exciton dissociation to charge migration, charge trapping and charge coalescence to indirect excitons as a function of the excitation density in a multilayer TMDC. We consider two aqueous dispersions of $\mathrm{MoS}_{2}$ with average layer number of $\langle N\rangle=2.5$ and $\langle N\rangle=9$ layers, respectively, obtained from LPE followed by cascade centrifugation for thickness selection ${ }^{20}$. We find that the primary photogenerated excitons dissociate into free charges, whose fate then depends on the sample thickness: in thin samples, bimolecular coalescence of charges creates indirect excitons, while in thick samples charges are efficiently trapped at local defects. The main difference between the two sample thicknesses is the defect content, which is higher by one order of magnitude in the thick sample, thus causing trapping to dominate over charge coalescence. 


\section{Results and discussion}

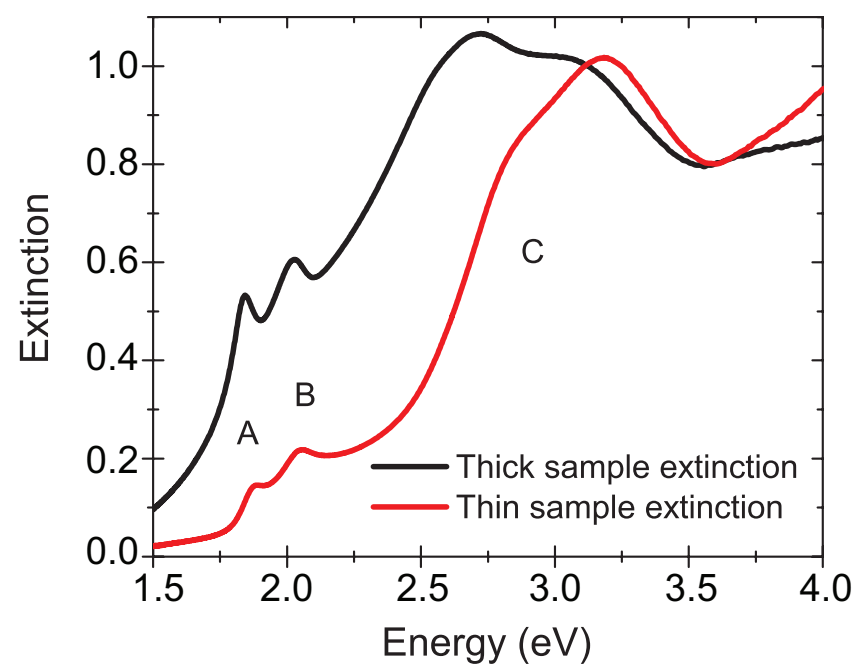

Figure 1 Extinction spectra of thin (red) and thick (black) $\mathrm{MoS}_{2}$ samples.

The extinction spectra of the studied samples, reported in Fig. 1, show three main peaks, which have been ascribed to absorption into the $\mathrm{A}, \mathrm{B}$, and $\mathrm{C}$ excitons ${ }^{1,2}$. Excitons $\mathrm{A}$ and $\mathrm{B}$ reside at the local band extrema at the $\mathrm{K}$ point, where the valence band $(\mathrm{VB})$ is split by spin-orbit coupling. The $\mathrm{C}$ exciton has been ascribed to a band nesting region around the $\Lambda$ (also called Q) point ${ }^{21-23}$. The detailed shape of the extinction spectrum provides a metric for the average number of layers $\langle N\rangle$ and the average lateral size $\langle L\rangle$ of an ensemble of flakes ${ }^{20} .\langle N\rangle$ is related to the exact position of the A peak, which is blue shifted for thinner flakes due to quantum confinement:

$$
<N>=2.3 \times 10^{36} e^{-44.26 \times E_{A}}
$$

Where $E_{\mathrm{A}}$ is the peak position, in $\mathrm{eV}$, of the $\mathrm{A}$ exciton in the extinction spectrum. Moreover, the ratio between the extinctions of the B exciton peak and the local minimum at $3.59 \mathrm{eV}\left(\frac{E x t_{\mathrm{B}}}{E x t_{3.59}}\right)$ changes with the lateral flake size $<L>$ :

$$
<L>(n m)=\frac{3.5 \frac{E x t_{\mathrm{B}}}{E x t_{3.59}}-0.14}{11.5-\frac{E x t_{\mathrm{B}}}{E x t_{3.59}}} \times 10^{-3},
$$

from these expressions we obtain $\langle N\rangle=9.7$ and $\langle L\rangle=170 \mathrm{~nm}$ for the thick and $\langle N\rangle=2.5$ and $<L>=70 \mathrm{~nm}$ for the thin flake ensemble.

We performed pump-probe spectroscopy with approximately 100 fs time resolution, using pump pulses at $3.1 \mathrm{eV}(400 \mathrm{~nm})$ and broad band probe pulses covering a spectral range from 1.65 to $2.85 \mathrm{eV}$. An energy of $50 \mathrm{~nJ}$ was set for the pump pulse, with a spot size of $140 \mu \mathrm{m}$ on the sample, resulting in a pump fluence of $8.12 \times 10^{-2} \mu \mathrm{J} / \mathrm{cm}^{-2}$. The $\mathrm{MoS}_{2}$ diferential transmission $\left(\frac{\Delta T}{T}\right)$ spectra, shown in Fig. 2A and D for the thick and the thin sample, respectively, display a series of alternating positive and negative features ${ }^{16,17}$. The customary interpretation of $\frac{\Delta T}{T}$ is based on the photoexcited depopulation of the ground state and population of excited states. The three main positive features spectrally coincide with the three main peaks of the extinction spectra. Such increased transmission is called photobleaching (PB) and corresponds to a weakening of the absorption from the ground state to the exciton states. Photoexcitation depletes the ground states of electrons available for the transitions and also populates possible final states of the transition, both of which reduces the absorption. Hence PB is generally an unspecific signature of photoexcited states; it indicates their presence but does not enable their unambiguous identification. Photoinduced absorption (PA) - negative $\frac{\Delta T}{T}$ - features, on the other hand, arise from transitions from photoexcited to higher excited states and are specific to the photoexcited species. 

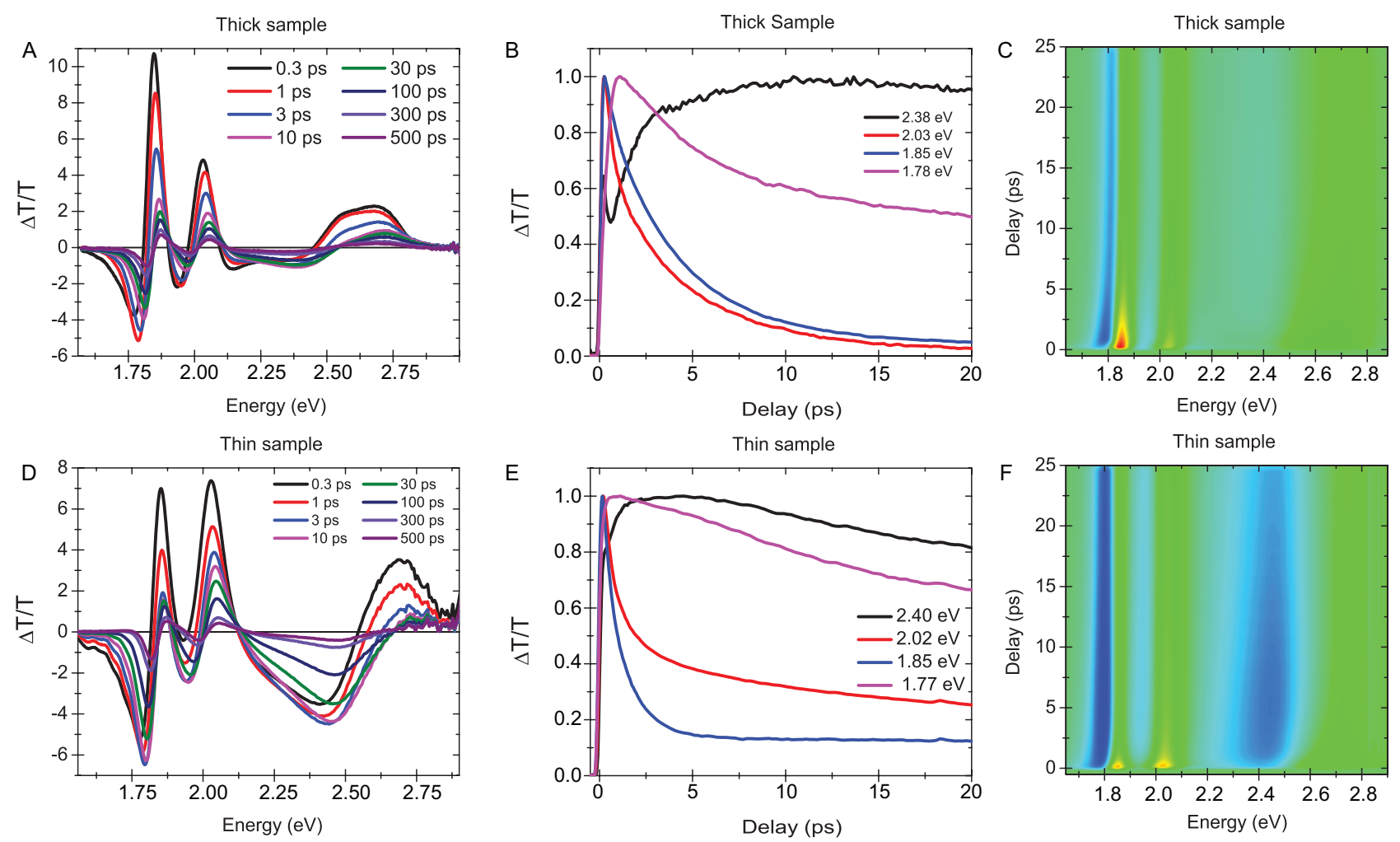

Figure $2(A),(D) \frac{\Delta T}{T}$ spectra at different delays for thick and thin sample respectively (B) thin sample's traces at 1.78(magenta), 1.85(cyan), 2.03 (red) and 2.38(black) (E) thick sample's traces at $1.77 \mathrm{eV}$ (magenta), $1.84 \mathrm{eV}$ (cyan), $2.02 \mathrm{eV}$ (red) and 2.38 (black) $(\mathrm{C}),(\mathrm{F}) \frac{\Delta T}{T}$ density map for thick and thin sample respectively

Recently, an alternative origin of $\frac{\Delta T}{T}$ during the first few 100 fs has been proposed ${ }^{24}$. Photoexcited carriers cause bandgap renormalization (BGR) - which red-shifts the absorption peaks - and lower the exciton binding energy through screening - which blue-shifts the absorption peaks. The combined action of these two opposing effects can result in a red or blue shift, which gives a first-derivative like shape for $\frac{\Delta T}{T}$, as has been observed in monolayer $\mathrm{MoS}_{2}$ at sub-ps pump-probe delays ${ }^{24}$. Due to the close vicinity of the A and B peaks, their first deriviative is a series of positive and negative peaks similar to our $\frac{\Delta T}{T}$ signal. Additionally, BGR and screening can also broaden the peaks ${ }^{13,25-27}$, leading to a second-derivative-like $\frac{\Delta T}{T}$, which again results in a series of positive and negative peaks. It has been found that the joint effects of screening and BGR are particularly strong during the first few hundreds of $\mathrm{fs}^{25}$, which corresponds to the relaxation of hot electron-hole pairs (e-h) into carriers at the band edges, while subsequently the spectrum is dominated by the more customary population effects $\mathrm{PB}$ and $\mathrm{PA}^{28}$.

The $\frac{\Delta T}{T}$ spectra evolve as follows. Photoexcitation yields a series of alternating PA and PB peaks, with strong $\mathrm{PB}$ features at the absorption resonances forming within the instrumental response. Over the next 1-3 ps, the PB decreases strongly and the PA continues to grow. For another few ps, all features decrease slowly and shift to the blue. Finally, the signal continues to decay without any significant shifts or changes in shape. Photoexcitation at $3.1 \mathrm{eV}$ creates an ensemble population of $\mathrm{C}$ excitons and hot carriers, which relax towards a different ensemble containing possibly A and B excitons and thermalized carriers on a time scale of approximately 1 ps $^{16-18,25,29,30}$ and subsequently evolve into further mixed ensemble populations of excitons and/or charges, as discussed below. We model this relaxation behaviour as a cascade ${ }^{29}$, where each step describes the evolution of one ensemble population of excited states into another. Since the interesting dynamics occurs in the first few tens of ps, we fit a temporal window from 0 to 20 ps using the following 
cascade:

$$
\begin{aligned}
\frac{d E(t)}{d t} & =G(t)-k_{1} E(t) \\
\frac{d C(t)}{d t} & =k_{1} E(t)-k_{2} C(t) \\
\frac{d F(t)}{d t} & =k_{2} C(t) \\
\frac{d G S(t)}{d t} & =-G(t)
\end{aligned}
$$

Where $G S$ is the ground state, $G(t)$ is the pump effect (which generates the starting photoexcited population). $E$ is the starting ensemble of photoexcited states that evolves with a rate constant $k_{1}$ into the second level of our cascade $(C) . C$ is populated only from $E$ and evolves into $F$ with a rate constant $k_{2}$. As mentioned above, the three ensembles may consist of different photoexcited state populations. The initial ensemble $\mathrm{E}$ consists of $\mathrm{C}$ excitons and hot carriers, while the composition of the subsequent ensembles will be discussed in the following. As at the end of our delay window we still have signal we can assume that there are some slower dynamics than the ones which are in the focus of this work. Level $F$ - the final ensemble in our model - will be the starting population for those slower processes.

We now present a model that relates the evolution of the $\Delta T / T$ spectra to the photoexcitation dynamics. According to Beer-Lambert's law, the transmittance through the sample is ${ }^{31}$ :

$$
T=e^{-\alpha(\omega) d}
$$

where $\alpha(\omega)$ is the frequency-dependent absorption coefficient and $\mathrm{d}$ is the sample thickness. It can easily be shown that, for $\Delta T / T \ll 1$ :

$$
\frac{\Delta T}{T}=-\Delta \alpha(\omega, t) d
$$

The absorption coefficient can be written as $\alpha(\omega, t)=n(t) \sigma(\omega, t)$, where $\mathrm{n}$ is the density of absorbers and $\sigma(\omega, t)$ is the is their absorption cross section. This can be easily translated to a change in the transmitted light.

$$
\frac{\Delta T}{T}(\omega, t)=-\sum_{i}\left[\sigma_{i}(\omega) \Delta n_{i}(t)+n_{i}(t) \Delta \sigma_{i}(\omega, t)\right]
$$

where $i$ denotes the ground state and the various excited state populations. The first term reflects the photoinduced changes in the populations. The second term represents changes in the absorption spectra as a consequence of photoexcitation, such as band gap renormalization, ${ }^{24}$ the Burstein-Moss effect, ${ }^{34}$ or Stark effect due to photoexcited charges. ${ }^{35-37}$ All these effects are population dependent, so that the time dependence of $\sigma_{i}(\omega, t)$ also follows the time dependence of the photoexcited populations. In many cases the second term is either negligible or indistinguishable from a contribution to the first term- since it has the same time dependence as $\Delta n$-, and the data can be modeled using only the first term of Eq 9 with a time-independent $\sigma(\omega) .{ }^{38}$

We fit the temporal evolution of $\Delta \mathrm{T} / \mathrm{T}$ using Eq. 9, using the first term only, and fitting the time dependent ensemble populations according to the model in Eqs. 3-6 and obtain $k_{1}=1 \mathrm{ps}^{-1}$ and $k_{2}=0.045$ $\mathrm{ps}^{-1}\left(\tau_{1}=1 \mathrm{ps}\right.$ and $\left.\tau_{2}=22 \mathrm{ps}\right)$ for the thin and $k_{1}=2.5 \mathrm{ps}^{-1}$ and $k_{2}=0.31 \mathrm{ps}^{-1}\left(\tau_{1}=0.4 \mathrm{ps}\right.$ and $\tau_{2}$ $=3.2 \mathrm{ps}$ ) for the thick sample. In few-layer $\mathrm{MoS}_{2}, k_{1}$ has been attributed to the relaxation ("cooling") of hot e-h pairs towards relaxed charge carriers ${ }^{16,17,25} . k_{2}$ is less well studied; in a previous study on $\mathrm{WS}_{2}$ dispersed in PMMA films, it was assigned to carrier diffusion until they are trapped ${ }^{29}$. Rates very similar to our $k_{2}$ have been observed before and assigned to trap-mediated charge recombination ${ }^{32}$, exciton-exciton annihilation ${ }^{19}$, exciton recombination ${ }^{18}$, relaxation of optical phonons ${ }^{17}$ or carrier-phonon scattering ${ }^{33}$. 

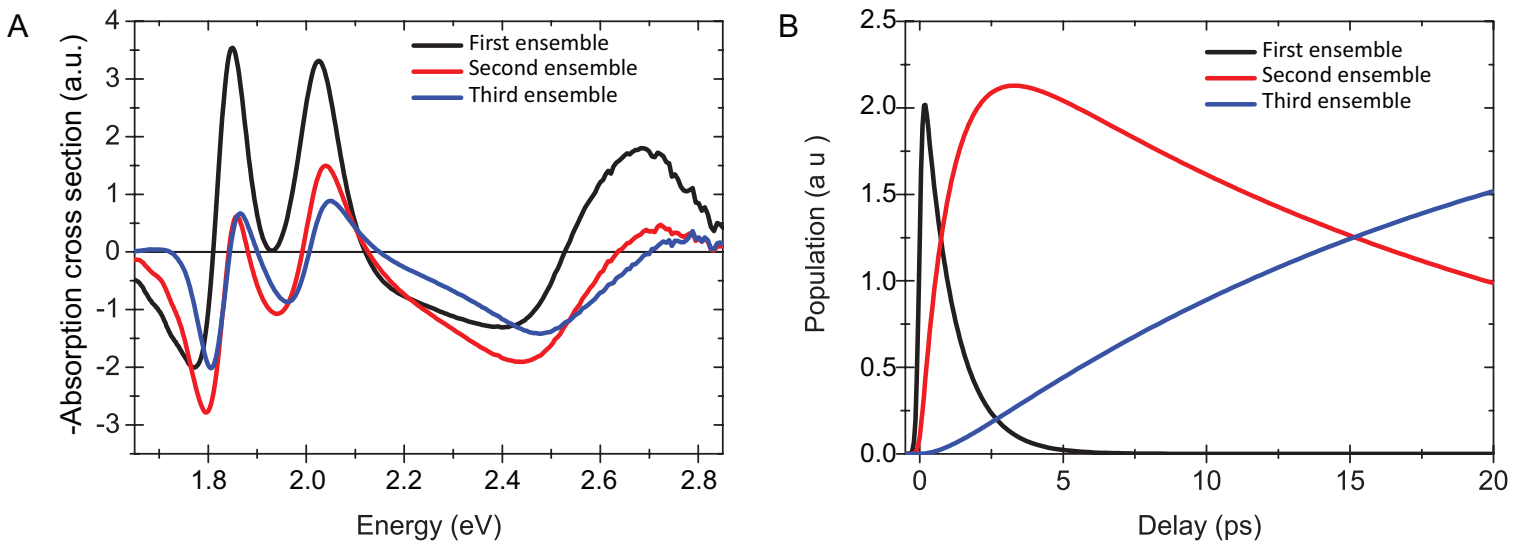

Figure 3 (A)Spectral contribution of each cascade level to the pump probe spectrum of thin sample pumped at $50 \mu \mathrm{W}$ (B) Evolution of the population of each state in thin sample when pumped at $50 \mu \mathrm{W}$.

The spectra of the ensemble populations at the various stages of the cascade are shown in Fig. 3A. The first population are the hot e-h pairs whose main spectral signature is the derivative-like $\frac{\Delta T}{T}$ due to shifts and broadenings of the absorption peaks. The two subsequent spectra are rather similar in shape and magnitude. This small spectral change does not suggest an evolution between different photoexcited species. It is more plausible that the two ensemble populations consist of the same photoexcited populations, with different time dependent environmental influence, such as an evolution from free to trapped charge carriers.

The band structure of $\mathrm{MoS}_{2}$ and similar TMDCs shows a characteristic evolution with the number of layers ${ }^{1,2,39,40}$. While in the monolayer the VB maximum and the conduction band (CB) minimum are both at the $\mathrm{K}$ point, in the bilayer and all thicker samples the VB maximum is at $\Gamma$ and the $\mathrm{CB}$ minimum is at a point $\Lambda$ between $\Gamma$ and $K$. Hence, from the bilayer onwards the energy minima for electrons are at the same points in the band structure, which suggests that both in our thin and thick sample the same energy relaxation pathways for photoexcited excitons and charges should exist. However, the relaxation rates along the different possible pathways, and hence which of them dominates the observed cascade, may be different due to different exciton binding energies or different defect content.

To gain further insight into the interaction of charges and excitons with each other and with possible traps, we repeat the above described measurements and analysis for excitation at different fluences, from 50 to $420 \mu W$ (which corresponds to a range of density of photoexcited states from $2.4 \times 10^{11}$ to $7.4 \times 10^{12}$ photoexcited states per $\left.\mathrm{cm}^{2}\right)$.

$k_{1}$ decreases as pump power increases for both samples (Fig S5), which is in agreement with a cooling behavior ${ }^{17}$. Both samples behave similarly and with time constant values in a similar range, that would indicate that photoexcited e-h pairs dissociate during cooling. The decreased cooling rate for higher excitation densities may be due to a phonon bottleneck ${ }^{41}$.

For the thick sample $k_{2}$ decreases slightly upon increasing the fluence (See Fig. 4), by one third over an almost tenfold increase of the fluence. As mentioned above, the slight change of the spectrum without significant reduction of the oscillator strength from the middle to the final ensemble in the cascade suggests that the process described by the rate constant $k_{2}$ corresponds to diffusion and trapping. The slowing down of this process with increasing fluence suggests saturation of traps. As traps are filled with charges, the free charges need to travel longer distances to encounter a trap that is still free.

For the thin sample, on the other hand, the rate constant $k_{2}$ increases with increasing fluence. Such behaviour points either towards the recombination of an electron and a hole towards the ground state or their coalescence to form an (A, B, or indirect) exciton. Previously, recombination has been discussed for excitons in monolayer $\mathrm{MoS}_{2}{ }^{19}$ and $\mathrm{WS}_{2}{ }^{42}$. In our sample with $\langle N\rangle=2.5$, photoexcitation with a large excess energy yields charge carriers rather than excitons ${ }^{22}$. Hence $k_{2}$ is a process that happens to charges. Charge recombination and coalescence can be geminate or non-geminate. In the geminate case the electron and hole are from the same originally excited hot e-h pair. This process is independent of the presence of other charges (unless the charge density becomes high enough to significantly screen the e-h interaction) and should have a rate $k_{2}$ independent of the excitation density. In the non-geminate case, the electron recombines with a hole that originates from a different exciton. If the rate limiting step of this process is the 


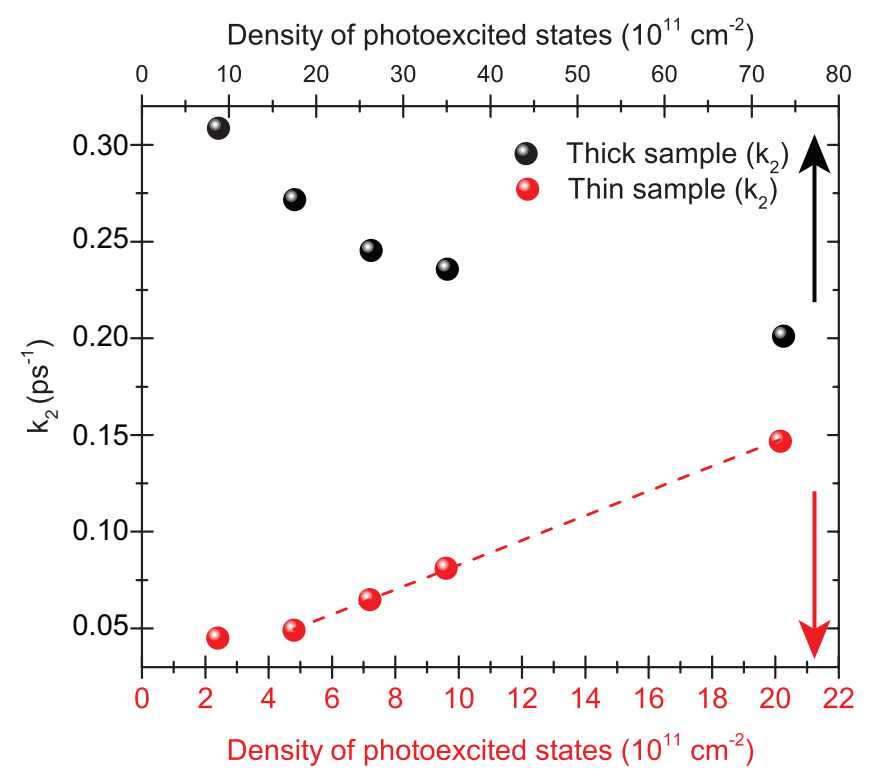

Figure $4 k_{2}$ density of photoexcited states dependence for thin and thick sample.

recombination itself, then the electron population decays as:

$$
\frac{d e}{d t}=-\gamma e h
$$

with $e$ and $h$ being the electron and hole densities and $\gamma$ the annihilation parameter. Since optical excitation generates electrons and holes in pairs, $e=h$, and equation 10 can be rewritten as:

$$
\frac{d e}{d t}=-\gamma e^{2}=-\gamma h^{2}
$$

If such a process is fitted approximately with an exponential decay, the rate constant $k_{2}$ will be proportional to $\gamma h$ and hence proportional to the excitation density. The fluence dependent $k_{2}$ shown in Fig. 4 is linear in the fluence, but does not go to zero for zero fluence. We thus conclude that, for the used fluences (with the possible exception of the lowest one), there is a strong non-geminate charge recombination or coalescence occurring together with a weaker contribution of a mostly intensity-independent process, either geminate recombination/coalescence or trapping without saturation.

Among the possible excited species evolution reported for $\mathrm{MoS}_{2}$, charges may coalesce to form A or B excitons, indirect excitons, or recombine and directly repopulate the ground state. If they coalesced into A and/or B excitons, the spectrum of the third ensemble in Fig. 3a should look more different from the second one, in particular with less PA from charges. If they recombined towards the ground state, the spectrum of the third state should ideally be zero. The remaining and most plausible scenario is therefore coalescence into indirect excitons. After exciton dissociation, the initially hot electrons and holes relax towards the minimum of the conduction band and the maximum of the VB, respectively, which are at different momenta due to the indirect bandgap of the multilayer TMDC and result in the formation of indirect excitons.

Both indirect excitons and trapped charges are long lived. After a few tens of picoseconds the $\frac{\Delta T}{T}$ signal decays without further significant changes in the spectral shape. Hence there is a decay channel towards the ground state. The rate of that decay seems to slow down with increasing delay. In fact, in a similar sample dispersed in PMMA, a photoexcited $\frac{\Delta T}{T}$ signal originating from long-lived states has been observed even on the ms time scale ${ }^{16}$.

The main difference between the $\langle N\rangle=2.5$ and $\langle N\rangle=9$ samples is that for the thinner flakes non-geminate coalescence into indirect excitons is the dominant charge carrier relaxation process with the rate $k_{2}$, while for the thicker flakes it is trapping of charges. Following this assignment for the rate $k_{2}$, we model the intensity dependent dynamics in order to determine the trap density. To this end, we replace Eq. 4 with 


$$
\frac{d C(t)}{d t}=k_{1} E(t)-\gamma C^{2}(t)-\gamma C(t) N_{T}(t)
$$

Where $\gamma$ is the rate parameter of bimolecular recombination and $N_{T}(t)$ is the time dependent density of active traps. Since recombination and trapping are both related to diffusion, we assume that they have the same rate parameter. Hence, both processes are active i both samples, but recombination dominates for $C \gg N_{T}$ and trapping for $C \ll N_{T}$. Traps are active only when they are not occupied, hence trapping of a charge deactivates one trap:

$$
\frac{d T(t)}{d t}=-\gamma C(t) T(t)
$$

When fitting the dynamics for the five different intensities with the same parameters, we obtain $\gamma=$ $4 \times 10^{-13} \mathrm{~cm}^{2} \mathrm{~s}^{-1}$ and $N_{T}(0)=1.5 \times 10^{12} \mathrm{~cm}^{-2}$ for the thick and $\gamma=1.5 \times 10^{-13} \mathrm{~cm}^{2} \mathrm{~s}^{-1}$ and $N_{T}(0)=1.6 \times 10^{11}$ $\mathrm{cm}^{-2}$ for the thin sample. Both these trap densities as well as the time scale agree well with a previous study on monolayer $\mathrm{MoS}_{2}{ }^{32}$ and with surface energy measurements in $\mathrm{LPE} \mathrm{MoS}_{2}{ }^{43}$ Since $\gamma$ is related to the diffusion constant, which in turn is proportional to the charge mobility via the Einstein relation, our result suggests a higher charge mobility in the thicker flake. This is in good agreement with previous studies, which showed that the highest mobility values are obtained for $\langle N\rangle=7-9$, and the mobility for $\langle N\rangle=2.5$ is $\sim 4.5$ times lower ${ }^{44-46}$. The trap density in the thicker sample is about one order of magnitude higher than in the thin one. Defects in TMDCs, such as Sulphur vacancies or additional metal atoms have been studied in detail with transmission electron and scanning tunneling microscopy (TEM, STM) as well as modelled with density functional theory. ${ }^{47-50}$ Typical defect densities exceed $10^{13} \mathrm{~cm}^{-2}, 50$ which is higher than the density $\left(10^{11}-10^{12} \mathrm{~cm}^{-2}\right)$ of traps for charges or excitons observed in electrical and spectroscopic measurements. ${ }^{32,51,52}$ As a result of this discrepancy, the exact atomic origin of these traps has not yet been identified and is beyond the scope of this paper. However, we can still gain some insight into the origin of the different observed trap densities. Our thin and thick flakes originate from the same fabrication process; only in the last step the different thicknesses are selected via fractional centrifugation. This suggests two possible scenarios, either the trap density correlates with thickness or the traps in thicker flakes are more effective. The first scenario may be related to the LPE process. Domains of the microcrystalline starting material with a low trap density are closer to the ideal Van der Waals crystal that should exfoliate easily, while in domains with a higher trap content the layers may hold more strongly together and will exfoliate only into thicker flakes. This would be in agreement with a reported change in the photoluminescence full width half maximum as a function of particle size. ${ }^{53}$ The second scenario may be related to the interaction of the flakes with the solvent and surfactant. Traps can be screened by dielectrics. ${ }^{3,54}$ Solutions of ionic surfactants have high dielectric constants even at modest concentrations. ${ }^{55}$ Indeed, $N_{T}(0)$ obtained for our thick flakes is consistent with the values obtained from samples with no or weak screening, while $N_{T}(0)$ for the thin flakes agrees with the values found for efficient screening. Hence, traps at the flake surface may be screened efficiently, while those inside the thicker flakes remain active.

\section{Conclusion}

From our results a unified picture of photoexcitation dynamics in multilayer $\mathrm{MoS}_{2}$ emerges. Photoexcitation generates hot electron-hole pairs as the primary photoexcited species. These relax on the picosecond timescale and form charge carriers at the band edges. For low excitation densities, these charges diffuse until they are trapped at defects such as sulphur vacancies. For excitation densities exceeding the trap density, non-geminate coalescence into indirect excitons is the dominant process. The intensity dependent relaxation behaviour of the $\frac{\Delta T}{T}$ signal may thus provide insight into the trap density of a sample, allowing e.g. for systematic studies of the growth or exfoliation processes.

\section{Experimental section}

Sample preparation: Samples were obtained by liquid phase exfoliation (LPE) ${ }^{4,20,56-58}$. Measurements were done in aqueous dispersion because even though it is possible to produce solid TMDCs-polymer composites $^{59}$, during the drying process there is some partial reaggregation that is undesirable for this study. 
$\mathrm{MoS}_{2}$ was purchased from Sigma Aldrich at the highest available purity and used as received. Liquid exfoliated $\mathrm{MoS}_{2}$ was obtained by mixing $\mathrm{MoS}_{2}$ powder (initial concentration $\mathrm{C}_{i}=30 \mathrm{~g} / \mathrm{L}$ ) with a watersodium cholate dispersion (starting sodium cholate concentration $\mathrm{C}_{S C}=8 \mathrm{~g} / \mathrm{L}$ ). $80 \mathrm{~mL}$ of this mixture was sonicated, using a Cole Palmer 750W ultrasonic processor, CV33 probe head with a step horn 1/2 solid tapered $630-0219$ tip, during one hour at $65 \%$ of power.

This dispersion was afterwards centrifuged at an acceleration of $2700 \mathrm{~g}$ for 90 minutes. Supernatant was discarded and sediment was redispersed in a $2 \mathrm{~g} / \mathrm{L}$ concentration water-sodium cholate dispersion. This mixture was exfoliated for 5 hours using $65 \%$ of power. During the whole exfoliation procedure the sample was cooled by ice bath.

After this exfoliation procedure we have heterogeneous flakes dispersion (Stock dispersion) in terms of lateral length and number of layers. Final samples are size selected by centrifugation steps of 90 minutes.

Stock dispersion was centrifuged at $240 \mathrm{~g}$. Sediment was discarded due to its high content of nonexfoliated material. Supernatant was centrifuged at $840 \mathrm{~g}$, sediment was redispersed in $2 \mathrm{~g} / \mathrm{L}$ water-sodium cholate dispersion, resulting in our thick sample. Supernatant was centrifuged at $4800 \mathrm{~g}$. Sediment was discarded and supernatant was centrifuged at $8600 \mathrm{~g}$. Sediment was redispersed in a $2 \mathrm{~g} / \mathrm{L}$ water-sodium cholate dispersion and used as our thin sample.

This protocol has been proved to be useful for selecting the samples in terms of thickness and lateral $\operatorname{size}^{20}$. From the A exciton position and the ratio between the B exciton and the local minimum at $345 \mathrm{~nm}$ in the extinction spectra it is possible to estimate $\langle L\rangle$ and $\langle N\rangle$ of each sample.

Femtosecond pump-probe measurements: The femtosecond pump-probe spectroscopy set-up is driven by an amplified Ti:sapphire laser (Coherent Libra) producing $4 \mathrm{~mJ}, 100 \mathrm{fs}, 1.55 \mathrm{eV}$ pulses at 1-kHz repetition rate. A fraction of the pulse energy is frequency doubled in a beta-barium borate crystal, obtaining the $3.1 \mathrm{eV}$ photon energy pump pulses. Another fraction of the pulse energy is focused in a $3 \mathrm{~mm}$ thick sapphire plate to generate a single-filament white light continuum used as a probe. Pump and probe are non-collinearly focused (spot diameter $\sim 150 \mu \mathrm{m}$ ) on the sample and the transmitted probe spectrum is detected by a spectrometer working at the full $1-\mathrm{kHz}$ repetition rate of the laser. $\frac{\Delta T}{T}$ spectra are recorded with a time resolution of $\sim 100 \mathrm{fs}$ and a sensitivity of $1-2 \times 10^{-5}$

Before pump-probe measurements the concentration of the sample was adjusted in order to have an optical density of 1 at the pump wavelength.

Extinction measurements: Dispersions were measured using Hellma Analytics cuvette with 2mm optical path. A Perkin Elmer Lambda 1050 UV-Visible spectrometer was used to acquire extinction and absorption spectra (see supplementary information).

\section{Acknowledgement}

The research leading to these results has received funding from the Marie-Curie ITN "MoWSeS" (grant no. 317451), the Slovenian Research Agency programme P1-0040 and European Research Council advanced grant TRAJECTORY.

\section{References}

[1] K. F. Mak, C. Lee, J. Hone, J. Shan, and T. F. Heinz, "Atomically thin $\mathrm{MoS}_{2}$ : A new direct-gap semiconductor," Phys. Rev. Lett., vol. 105, no. 13, p. 136805, 2010.

[2] A. Splendiani, L. Sun, Y. Zhang, T. Li, J. Kim, C.-Y. Chim, G. Galli, and F. Wang, "Emerging photoluminescence in monolayer $\mathrm{MoS}_{2}$," Nano Lett., vol. 10, no. 4, pp. 1271-1275, 2010.

[3] B. Radisavljevic, A. Radenovic, J. Brivio, V. Giacometti, and A. Kis, "Single-layer $\mathrm{MoS}_{2}$ transistors," Nature Nanotech., vol. 6, no. 3, pp. 147-150, 2011.

[4] V. Nicolosi, M. Chhowalla, M. G. Kanatzidis, M. S. Strano, and J. N. Coleman, "Liquid exfoliation of layered materials," Science, vol. 340, no. 6139, p. 1226419, 2013.

[5] M. Amani, D.-H. Lien, D. Kiriya, J. Xiao, A. Azcat, J. Noh, S. R. Madhvapathy, R. Addou, S. KC, M. Dubey, K. Cho, R. M. Wallace, S.-C. Lee, J.-H. He, J. W. A. III, X. Zhang, E. Yablonovitch, and A. Javey, "Near-unity photoluminescence quantum yield in $\mathrm{MoS}_{2}$," Science, vol. 350, no. 6264, pp. 1065-1068, 2015. 
[6] B. Radisavljevic and A. Kis, "Mobility engineering and a metal-insulator transition in monolayer $\mathrm{MoS}_{2}$," Nat. Mater., vol. 12, no. 9, pp. 815-820, 2013.

[7] D. Costanzo, A. Jo, H. Berger, and A. F. Morpurgo, "Gated induced superconductivity in atomically thin $\mathrm{MoS}_{2}$ crystal," Nature Nanotech., vol. 11, no. 4, pp. 339-344, 2015.

[8] Y. Gong, J. Lin, X. Wang, G. Shi, S. Lei, Z. Lin, X. Zou, G. Ye, R. Vajtai, B. I. Yakobson, H. Terrones, M. Terrones, B. K. Tay, J. Lou, S. T. Pantelides, Z. Liu, W. Zhou, and P. M. Ajayan, "Gated induced superconductivity in atomically thin $\mathrm{MoS}_{2}$ crystal," Nature Mater., vol. 13, pp. 1135-1142, 2014.

[9] M. Massicotte, P. Schmidt, F. Vialla, K. G. Schadler, A. Reserbat-Plantey, K. Watanabe, T. Taniguchi, K. J. Tielrooij, and F. H. L. Koppens, "Picosecond photoresponse in Van der Waals heterostructures," Nat. Nanotech., vol. 11, no. 1, pp. 42-46, 2015.

[10] M. Buscerna, J. O. Island, D. J. Groenendijk, S. I. Blanter, G. A. Steele, H. S. J. V. der Zant, and A. Castellanos-Gomez, "Photocurrent generation with two-dimensional Van der Waals semiconductors," Chem. Soc. Rev, vol. 44, no. 11, pp. 3691-3718, 2015.

[11] A. G. Kelly, T. Hallam, C. Backes, A. Harvey, A. S. Esmaeily, I. Godwin, J. Coelho, V. Nicolosi, J. Lauth, A. Kulkarni, S. Kinge, L. D. A. Siebbeles, G. S. Duesberg, and J. N. Coleman, "All-printed thin-film transistors from networks of liquid-exfoliated nanosheets," Science, vol. 356, no. 6333, pp. 69-73, 2017.

[12] Z. M. Wang, $M_{0} S_{2}$ Materials, Physics, and Devices. Springer Berlin Heidelberg New York: Springer, 2014.

[13] D. Vella, D. Ovchinnikov, N. Martino, V. Vega-Mayoral, D. Dumcenco, Y.-C. Kung, M.-R. Antognazza, A. Kis, G. Lanzani, D. Mihailovic, and C. Gadermaier, "Unconventional electroabsorption in monolayer $\mathrm{MoS}_{2}, " 2 D$ Mater, vol. 4, no. 2, p. 021005, 2017.

[14] J. Zeng, M. Yuan, W. Yuan, Q. Dai, H. Fan, S. Lan, and S. Tie, "Enhanced second harmonic generation of $\mathrm{MoS}_{2}$ layers on a thin gold film," Nanoscale, vol. 7, pp. 13547-13553, 2015.

[15] Y. Ye, J. Wong, X. Lu, X. Ni, H. Zhu, X. Chen, Y. Wang, and X. Zhang, "Monolayer excitonic laser," Nature Photon., vol. 9, pp. 733-737, 2015.

[16] T. Borzda, C. Gadermaier, N. Vujicic, P. Topolovsek, M. Borovsak, T. Mertelj, D. Viola, C. Manzoni, E. A. A. Pogna, D. Brida, M. R. Antognazza, F. Scotognella, G. Lanzani, G. Cerullo, and D. Mihailovic, "Charge photogeneration in few-layer $\mathrm{MoS}_{2}$, , Adv. Mater., vol. 25, no. 22, pp. 3351-3358, 2015.

[17] Z. Nie, R. Long, L. Sun, C.-C. Huang, J. Zhang, Q. Xiong, D. W. Hewak, Z. Shen, O. V. Prezhdo, and Z.H. Loh, "Ultrafast carrier thermalization and cooling dynamics in few-layer $\mathrm{MoS}_{2}$," ACS Nano, vol. 8, no. 10, pp. 10931-10940, 2014.

[18] P. D. Cunningham, K. M. McCreary, A. T. Hanbicki, M. Currie, B. T. Jonker, and L. M. Hayden, "Charge trapping and exciton dynamics in large -area CVD grown $\mathrm{MoS}_{2}$," J. Phys. Chem. C, vol. 120, no. 10, pp. 5819-5826, 2016.

[19] D. Sun, Y. Rao, G. A. Reider, G. Chen, Y. You, L. Breezin, A. R. Harutyunyan, and T. F. Heinz, "Observation of rapid exciton-exciton annihilation in monolayer molybdenum disulfide," Nano Lett., vol. 14, no. 10, pp. 5625-5629, 2014.

[20] C. Backes, R. J. Smith, N. McEvoy, N. C. Berner, D. McCloskey, H. C. Nerl, A. O’Neill, P. J. King, T. Higgins, D. Hanlon, N. Scheuschner, J. Maultzsch, L. Houben, G. S. Duesberg, J. F. Donegan, V. Nicolosi, and J. N. Coleman, "Edge and confinement effects allow in situ measurement of size and thickness of liquid-exfoliated nanosheets," Nat. Commun., vol. 5, p. 4576, 2014.

[21] A. Kormanyos, G. Burkard, M. Gmitra, J. Fabian, V. Zolyomi, N. D. Drummond, and V. Fal'ko, "k p theory for two-dimensional transition metal dichalcogenide semiconductors," 2D Matter, vol. 2, p. 022001, 2015. 
[22] D. Kozawa, R. Kumar, A. Carvalho, K. K. Amara, W. Zhao, S. Wang, M. Toh, R. M. Ribeiro, A. C. Neto, K. Matsuda, and G. Eda, "Photocarrier relaxation pathway in two-dimensional semiconducting transition metal dichalcogenides," Nature Commun., vol. 5, p. 4543, 2014.

[23] R. Gillen and J. Maultzsch, "Light-matter interactions in two-dimensional transition metal dichalcogenides: Dominant excitonic transitions in mono- and few layer MoX $\mathrm{X}_{2}$ and band nesting," IEEE J. Sel. Top. Quantum Electron., vol. 23, no. 1, p. 9000512, 2017.

[24] E. A. A. Pogna, M. Marsili, D. de Fazio, S. D. Conte, C. Manzoni, D. Sangalli, D. Yoon, A. Lombardo, A. C. Ferrari, A. Marini, G. Cerullo, and D. Prezzi, "Photo-induced bandgap renormalization governs the ultrafast response of single-layer $\mathrm{MoS}_{2}$," ACS Nano, vol. 10, no. 1, pp. 1182-1188, 2016.

[25] D. Tsokkou, X. Yu, K. Sivula, and N. Banerji, "The role of excitons and free charges in the excitedstate dynamics of solution-processed few-layer $\mathrm{MoS}_{2}$ nanoflakes," J. Phys. Chem. C, vol. 120, no. 40, pp. 23286-23292, 2016.

[26] C. Ruppert, A. Chernikov, H. M. Hill, A. F. Rigosi, and T. F. Heinz, "The role of electronic and phononic excitation in the optical response of monolayer $\mathrm{WS}_{2}$ after ultrafast excitation," Nano Lett, vol. 17, no. 2, pp. 644-651, 2017.

[27] A. Chernikov, A. M. van der Zande, H. M. Hill, A. F. Rigosi, A. Velauthapillai, J. Hone, and T. F. Heinz, "Electrical tuning of exciton binding energies in monolayer $\mathrm{WS}_{2}$," Phys. Rev. Lett., vol. 115, no. 12, p. 126802, 2015.

[28] E. A. A. Pogna et al, "Quasiparticles versus many-body interactions in photoexcited $\mathrm{WS}_{2}$ nanoinks," to be submitted.

[29] V. Vega-Mayoral, D. Vella, T. Borzda, M. Prijatelj, Z. G. Tempra, E. A. A. Pogna, S. D. Conte, P. Topolovsek, N. Vujicic, G. Cerullo, D. Mihailovic, and C. Gadermaier, "Exciton and charge carrier dynamics in few-layer WS 2 ," Nanoscale, vol. 8, no. 10, pp. 5428-5434, 2016.

[30] D. Vella, D. Ovchinnikov, D. Viola, D. Dumcenco, Y.-C. Kung, E. A. A. Pogna, S. D. Conte, V. VegaMayoral, T. Borzda, M. Prijatelj, D. D. Mihailovic, A. Kis, G. Cerullo, and C. Gadermaier, "Field-induced charge separation dynamics in monolayer $\mathrm{MoS}_{2}, " 2 d$ Mater, vol. 4, p. 035017, 2017.

[31] A. Beer, "Bestimmung der Absorption des rothen Lichts in farbigen Flüssigkeiten," Annalen der Physik und Chemie, vol. 86, no. 5, pp. 78-88, 1852.

[32] H. Wang, C. Zhang, and F. Rana, "Ultrafast dynamics of defect-assisted electron-hole recombination in monolayer $\mathrm{MoS}_{2}$," Nano Lett., vol. 15, no. 1, pp. 339-345, 2015.

[33] H. Shi, R. Yan, S. Bertolazzi, J. Brivio, B. Gao, A. Kis, D. Jena, H. G. Xing, and L. Huang, "Exciton dynamics in suspended monolayer and few-layer $\mathrm{MoS}_{2} 2 \mathrm{~d}$ crystals," ACS Nano, vol. 7, no. 2, pp. 10721080, 2013.

[34] Q. C. Sun, L. Yadgarov, R. Rosentsveig, S. G, R. Tenne, and J. L. Musfeldt, "Observation of a BursteinMoss shift in rhenium-doped $\mathrm{MoS}_{2}$ nanoparticles.," ACS Nano, vol. 7, no. 4, pp. 3506-3511, 2013.

[35] V. I. Klimov, S. A. Ivanov, J. Nanda, M. Achermann, I. Bezel, J. A. McGuire, and A. Piryatinski, "Singleexciton optical gain in semiconductor nanocrystals," Nature, vol. 447, no. 7143, pp. 441-446, 2007.

[36] J. Cabanillas-Gonzalez, T. Virgili, A. Gambetta, G. Lanzani, T. D. Anthopoulos, and D. M. de Leeuw, "Photoinduced transient Stark spectroscopy in organic semiconductors: A method for charge mobility determination in the picosecond regime," Phys. Rev. Lett., vol. 96, no. 10, p. 106601, 2006.

[37] C. Gadermaier, E. Menna, M. Meneghetti, W. J. Kennedy, Z. V. Vardeny, and G. Lanzani, "Long-lived charged states in single-walled carbon nanotubes," Nano Lett., vol. 6, no. 2, pp. 301-305, 2006.

[38] J. Cabanillas-Gonzalez, G. Grancini, and G. Lanzani, "Pump-probe spectroscopy in organic semiconductors: Monitoring fundamental processes of relevance in optoelectronics," Adv. Mater., vol. 23, no. 46, pp. 5468-5485, 2011. 
[39] A. Kuc, N. Zibouche, and T. Heine, "Influence of quantum confinement on the electronic structure of the transition metal sulfide TS 2 ," Phys. Rev. B, vol. 83, no. 24, p. 245213, 2011.

[40] W. Zhao, Z. Ghorannevis, L. Chu, M. Toh, C. Kloc, P.-H. Tan, and G. Eda, "Evolution of electronic structure in atomically thin sheets of $\mathrm{WS}_{2}$ and WSe 2 ," ACS Nano, vol. 7, no. 1, pp. 791-797, 2013.

[41] R. Heitz, H. Born, F. Guffarth, O. Stier, A. Schliwa, A. Hoffmann, and D. Bimberg, "Existence of a phonon bottleneck for excitons in quantum dots," Phys. Rev. B, vol. 64, p. 241305(R), 2001.

[42] L. Yuan and L. Huang, "Exciton dynamics and annihilation in $\mathrm{WS}_{2} 2 \mathrm{~d}$ semiconductors," Nanoscale, vol. 7, no. 16, pp. 7402-7408, 2015.

[43] I. Caffey, J. N Coleman et al., "Private comunication," , 2017.

[44] M.-W. Lin, I. I. Kravchenko, J. Fowlkes, X. Li, A. A. Puretzky, C. M. Rouleau, D. B. Geohegan, and K. Xiao, "Thickness-dependent charge transport in few-layer $\mathrm{MoS}_{2}$ field-effect transistors," Nanotechnology, vol. 27, p. 165203, 2016.

[45] S.-L. Li, K. Wakabayashi, Y. Xu, S. Nakaharai, K. Komatsu, W.-W. Li, Y.-F. Lin, A. Aparecido-Ferrera, and K. Tsukagoshi, "Thickness-dependent interfacial coulomb scattering in atomically thin field-effect transistors.," Nano. Lett, vol. 13, pp. 3546-3552, 2013.

[46] H. Schmidt, F. Giustiniano, and G. Eda, "Electronic transport properties of transition metal dichalcogenide field-effect devices: surface and interface effects," Chem. Soc. Rev., vol. 44, pp. 7715-7736, 2015.

[47] C. Ataka, H. Sahin, E. Akturk, and S. Ciraci, "Mechanical and electronic properties of MoS 2 nanoribbons and their defects," J. Phy. Chem. C, vol. 115, no. 10, pp. 3934-3941, 2011.

[48] W. Zhou, X. Zou, S. Najmaei, Z. Liu, Y. Shi, J. Kong, J. Lou, P. M. Ajayan, B. I. Yakobson, and J.-C. Idrobo, "Intrinsic structural defects in monolayer molybdenum disulfide," Nano Lett., vol. 13, no. 6, pp. 2615-2622, 2013.

[49] A. M. van der Zande, P. Y. Huang, D. A. Chenet, T. C. Berkelbach, Y. M. You, G.-H. Lee, T. F. Heinz, D. R. Reichman, D. A. Muller, and J. C. Hone, "Grains and grain boundaries in highly crystalline monolayer molybdenum disulphide," Nat Mater., vol. 12, no. 6, pp. 554-561, 2013.

[50] J. Hong, Z. Hu, M. Probert, K. Li, D. Lv, X. Yang, L. Gu, N. Mao, Q. Feng, L. Xie, J. Zhang, D. Wu, Z. Zhang, C. Jin, , W. Ji, X. Zhang, J. Yuan, and Z. Zhang, "Exploring atomic defects in molybdenum disulphide monolayers," Nature Comm., vol. 6, p. 6293, 2015.

[51] S. Tongay, J. Suh, C. Ataca, W. Fan, A. Luce, J. S. Kang, J. Liu, C. Ko, R. Raghunathanan, J. Zhou, F. Ogletree, J. Li, J. C. Grossman, and J. Wu, "Defects activated photoluminescence in two-dimensional semiconductors: interplay between bound, charged, and free excitons," Sci. Rep., vol. 3, p. 2657, 2013.

[52] M. M. Furchi, D. K. Polyushkin, A. Pospischil, and T. Mueller, "Mechanisms of photoconductivity in atomically thin $\mathrm{MoS}_{2}$," Nano Lett., vol. 14, pp. 6165-6170, 2014.

[53] C. Backes, B. M. Szydlowska, A. Harvey, S. Yuan, V. Vega-Mayoral, B. R. Davies, P. liang Zhao, D. Hanlon, E. J. G. Santos, M. I. Katsnelson, W. J. Blau, C. Gadermaier, and J. N. Coleman, "Production of highly monolayer enriched dispersions of liquid-exfoliated nanosheets by liquid cascade centrifugation," ACS Nano, vol. 10, no. 1, pp. 1589-1601, 2016.

[54] H. S. Song, S. L. Li, L. Gao, Y. Xu, K. Ueno, Y. B. Cheng, and K. Tsukagoshi, "High-performance topgated monolayer $\mathrm{SnS}_{2}$ field-effect transistors and their integrated logic circuits," Nanoscale, vol. 5, pp. 9666-9670, 2013.

[55] M. Abe and K. Ogino, "Dielectric constants and electrical conductivities of sodium dodecyl sulfate in aqueous solutions," J. Coll. Int. Sci, vol. 80, pp. 58-66, 1981. 
[56] Y. Hernandez, V. Nicolosi, M. Lotya, F. M. Blighe, Z. Sun, S. De, I. T. McGovern, B. Holland, M. Byrne, Y. K. Gun'Ko, J. J. Boland, P. Niraj, G. Duesberg, S. Krishnamurthy, R. Goodhue, J. Hutchison, V. Scardaci, A. C. Ferrari, and J. N. Coleman, "High-yield production of graphene by liquid-phase exfoliation of graphite," Nature Nanotech., vol. 3, no. 9, pp. 563-568, 2008.

[57] D. Hanlon, C. Backes, T. M. Higgins, M. Hughes, A. O’Neill, P. King, N. McEvoy, G. S. Duesberg, B. M. Sanchez, H. Pettersson, V. Nicolosi, and J. N. Coleman, "Production of molybdenum trioxide nanosheets by liquid exfoliation and their application in high-performance supercapacitors," Chem. Mater, vol. 26, no. 4, pp. 1751-1763, 2014.

[58] J. R. Brent, N. Savjani, E. A. Lewis, S. J. Haigh, D. J. Lewis, and P. O'Brien, "Production of few-layer phosphorene by liquid exfoliation of black phosphorus," Chem. Commun., vol. 50, no. 87, pp. 1333813341, 2014.

[59] V. Vega-Mayoral, C. Backes, D. Hanlon, U. Khan, Z. Gholamvand, M. O’Brien, G. S. Duesberg, C. Gadermaier, and J. N. Coleman, "Photoluminescence from liquid-exfoliated $\mathrm{WS}_{2}$ monomers in poly(vinyl alcohol) polymer composites," Adv. Funct. Mater., vol. 26, no. 7, pp. 1028-1039, 2016. 


\title{
Supplementary information for: Charge trapping and recombination in few layer $\mathrm{MoS}_{2}$
}

\author{
Victor Vega-Mayoral, ${ }^{* a, b, c}$ Tetiana Borzda, ${ }^{a, b}$ Daniele Vella, ${ }^{a, b, d}$ Matej Prijatelj,,${ }^{a, b}$ \\ Eva A. A. Pogna, ${ }^{e}$ Claudia Backes, ${ }^{c, f, g}$ Jonathan N Coleman, ${ }^{c, f}$ Giulio Cerullo, ${ }^{e}$ Dragan Mihailovic ${ }^{a, b, h}$ \\ and Christoph Gadermaier ${ }^{a, b, *}$ \\ *E-mail:vegamayv@tcd.ie \\ E-mail: christoph.gadermaier@ijs.si \\ ${ }^{a}$ Department of Complex Mater, Jozef Stefan Institute, Jamova cesta 39, 1000, Ljubljana, Slovenia \\ ${ }^{b}$ Jozef Stefan International Postgraduated School, Jamova cesta 39, 1000, Ljubljana, Slovenia \\ ${ }^{c}$ School of Physics, Trinity College Dublin Dublin 2, Ireland \\ ${ }^{d}$ Department of Physics, National University of Singapore, 2 Science Drive 3, Singapore 117542, Singapore \\ ${ }^{e}$ IFN-CNR, Department of Physics, Politecnico di Milano, P. Leonardo da Vinci 32, 20133 Milan, Italy \\ ${ }^{f}$ CRANN \& AMBER, Trinity College Dublin Dublin 2, Ireland \\ ${ }^{g}$ Applied Physical Chemistry department, Im Neuenheimer Feld 253, 69120 Heidelberg, Germany \\ ${ }^{h}$ Center of Excellence in Nanoscience and Nanotechnology, Jamova 39, 1000 Ljubljana, Slovenia
}




\section{Contents}

S1 Calculating the photoexcited states density 3

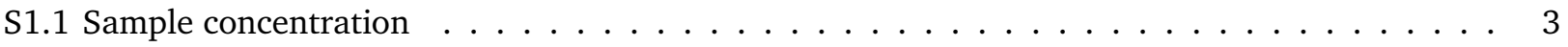

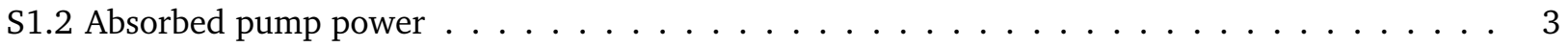

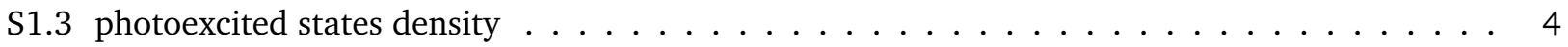

S1.3.1 Linear and non linear regime $\ldots \ldots \ldots \ldots \ldots \ldots$

$\begin{array}{lr}\text { S2 Calculating the trap density } & 6\end{array}$

$\begin{array}{ll}\text { S3 } k_{1} \text { thickness fluence dependence. } & 7\end{array}$

\section{List of Figures}

S1 Extinction and absorption spectra of thick (A) and thin (B) samples. . . . . . . . . . . 3

S2 $\mathrm{k} 2$ of thick and thin sample as a function of the $2 \mathrm{D}$ excitation density $\ldots \ldots \ldots \ldots$

S3 $\quad \frac{\Delta T}{T}$ signal intensity power dependence at $2.38 \mathrm{eV} \ldots \ldots \ldots \ldots \ldots$

S4 A and B exciton signal intensity as a function of the pump power $\ldots \ldots \ldots$

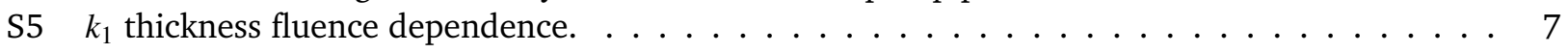




\section{S1 Calculating the photoexcited states density}

\section{S1.1 Sample concentration}

The Concentration of material in a dispersion is typically determined via filtration of the dispersion through a membrane. The mass of nanosheets is calculated by measuring the weight of the membrane before and after the filtration of a certain volume of dispersion. Some previous work on $\mathrm{MoS}_{2}$ developed a useful technique where the concentration can be calculated from the extinction spectra ${ }^{1}$. Then, the extinction coefficient and the concentration of dispersions can be obtained from their extinction spectra.

$$
\begin{aligned}
\varepsilon_{B} & =65.4-78.61 e^{-1.42796 x} \\
C & =\frac{E x t_{B}}{\varepsilon_{B} l}
\end{aligned}
$$

where $\varepsilon_{B}$ is the extinction coefficient at the $\mathrm{B}$ exciton, $\mathrm{x}$ is the ratio between the extinction at the $\mathrm{B}$ exciton and the local minimum at $3.59 \mathrm{eV}, \operatorname{Ext}_{B}$ is the extinction value at the B exciton resonance, $l$ is the light path of the cuvette $(0.2 \mathrm{~cm})$ and $C$ is the concentration of the dispersion. From this metric we have obtained a concentration of $0.08 \mathrm{~g} / \mathrm{L}$ for the thick sample and $0.09 \mathrm{~g} / \mathrm{L}$ for the thin sample.

\section{S1.2 Absorbed pump power}

In order to determine the fraction of light absorbed by the sample we will consider the absorption and not the extinction. From the Beer-Lambert law the absorbed power can be determined from:

$$
\begin{aligned}
P_{\text {transmitted }} & =P_{0} \times 10^{-A b s} \\
P_{A b s} & =P_{0}-P_{\text {transmitted }}
\end{aligned}
$$

A

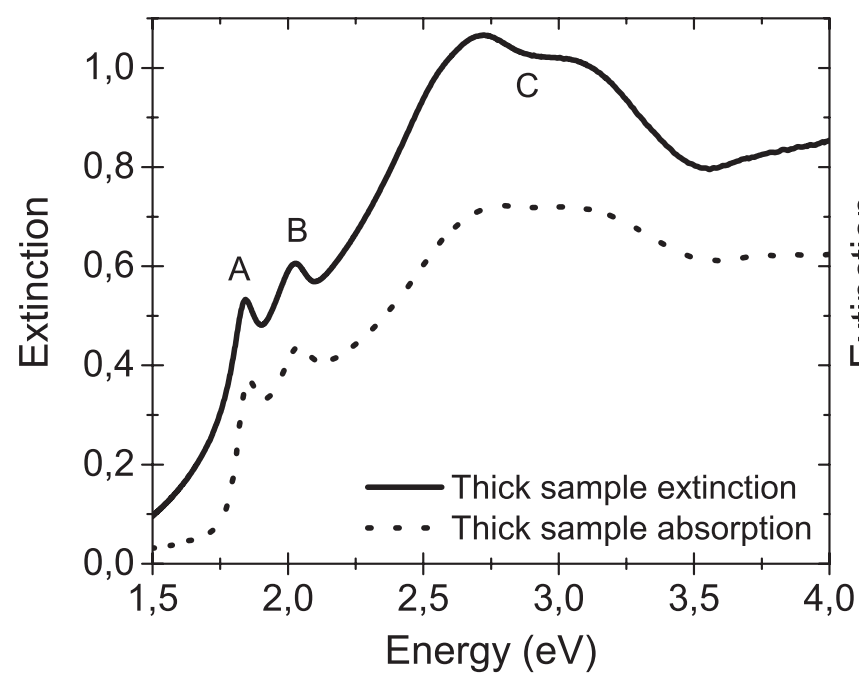

B

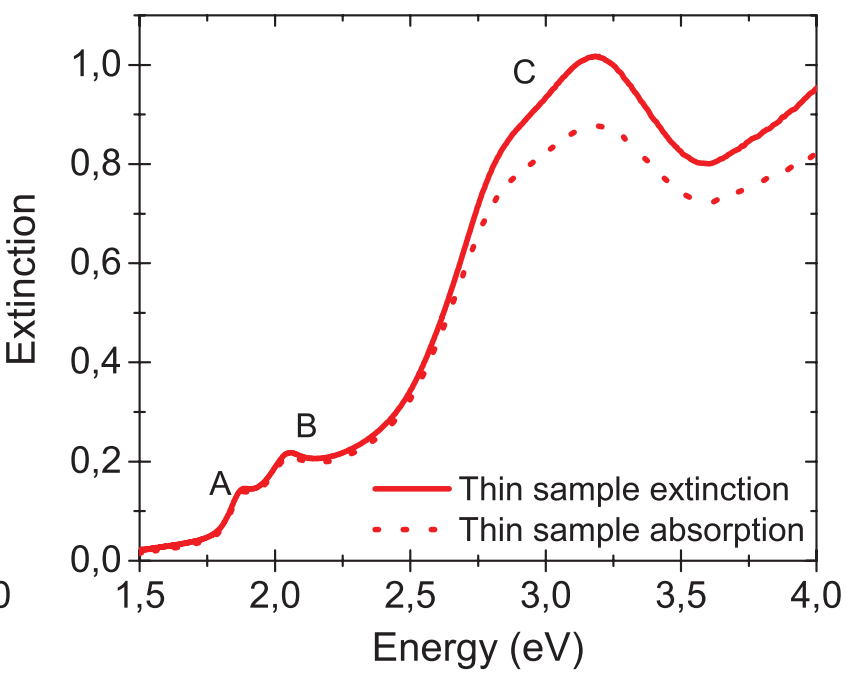

Figure S1 Extinction and absorption spectra of thick (A) and thin (B) samples.

As the absorption coefficient at the pump wavelength is 0.7 and 0.88 for the thick and thin sample respectively the chosen values of pump power will correspond to different absorbed power values in each sample. 


\begin{tabular}{|c|c|c|}
\hline Pump power $(\mu \mathrm{W})$ & Pump absorbed thick sample $(\mu \mathrm{W})$ & Pump absorbed thin sample $(\mu \mathrm{W})$ \\
\hline 50 & 40 & 43 \\
\hline 100 & 80 & 86 \\
\hline 150 & 120 & 130 \\
\hline 200 & 160 & 173 \\
\hline 420 & 336 & 364 \\
\hline
\end{tabular}

Table S1 Absorbed pump power for thick and thin sample

This difference comes from the different scattering contribution to the extinction spectra in both dispersions. The values of table S1 correspond to the absorbed light along $2 \mathrm{~mm}$ of dispersion. If we want to get the absorbed power in the region where pump and probe beams are overlapped, and we aproximate this region as an ellipsoid, we should scale the absorbance value:

$$
A^{\prime}=\frac{A * c}{l},
$$

where $c=500 \mu \mathrm{m}$ is the length of the ellipsoid and $l=0.2 \mathrm{~cm}$ is the length of the cuvette used in the extinction and absorption measurements. By doing so, we obtain the following absorbed power (table S2).

\begin{tabular}{|c|c|c|}
\hline Pump power $(\mu \mathrm{W})$ & Pump absorbed thick sample $(\mu \mathrm{W})$ & Pump absorbed thin sample $(\mu \mathrm{W})$ \\
\hline 50 & 16 & 19 \\
\hline 100 & 33 & 39 \\
\hline 150 & 49 & 59 \\
\hline 200 & 66 & 79 \\
\hline 420 & 139 & 166 \\
\hline
\end{tabular}

Table S2 Absorbed pump power for thick and thin sample in the pump and probe beam overlapping region.

\section{S1.3 photoexcited states density}

As we are dealing with 2D materials the photoexcited states density which is typically considered is the 2D excitation density. This is easier to calculate in a mechanically exfoliated sample, where a single flake is probed and the absorbed light can be measured directly. In order to obtain the photoexcited states density some calculation must be done. If we assume that we are probing an ellipsoid of radii a, b and c (150, 150 and $500 \mu \mathrm{m}$ respectively), and we know the concentration of our dispersions we can obtain the mass of material sampled

$$
\begin{aligned}
M & =C \times V_{\text {ellipsoid }} \\
V_{\text {ellipsoid }} & =\frac{4}{3} \pi a b c
\end{aligned}
$$

If we know the mass and the density of $\mathrm{MoS}_{2}\left(\rho_{\mathrm{MoS}_{2}}=5.06 \mathrm{~g} / \mathrm{L}\right)$ we can calculate the volume of material (not to be mistaken with the volume of the ellipsoid)

$$
V_{\text {material }}=\frac{M}{\rho_{\mathrm{MoS}_{2}}}
$$

As the average layer number of the flakes in dispersion has been calculated $(<N\rangle)$ and knowing the $\mathrm{MoS}_{2}$ single layer thickness $(\mathrm{d}=0.65 \mathrm{~nm})$ we can obtain the total surface tested:

$$
S=\frac{V_{\text {material }}}{<N>d}
$$


Taking the previous results in consideration, and knowing that the chosen laser has a repetition rate of $1 \mathrm{KHz}(f)$ and that we are pumping at $400 \mathrm{~nm}(3.1 \mathrm{eV})$ we can estimate the photoexcited states density as follows:

$$
\sigma_{\text {photoexcited states }}=\frac{P_{\text {Absorbed }}}{f \times E_{\text {photon }}}
$$

Applying Eq. 10 to the values of table S2 we can obtain the photoexcited states density used in Fig 4 of the main document:

\begin{tabular}{|c|c|c|}
\hline Pump power $(\mu \mathrm{W})$ & Thick sample $\left(10^{12} \mathrm{~cm}^{-2}\right)$ & Thin sample $\left(10^{12} \mathrm{~cm}^{-2}\right)$ \\
\hline 50 & 0.88 & 0.24 \\
\hline 100 & 1.75 & 0.48 \\
\hline 150 & 2.63 & 0.72 \\
\hline 200 & 3.50 & 0.95 \\
\hline 420 & 7.36 & 2.02 \\
\hline
\end{tabular}

Table S3 Photoexcited states density for thick and thin sample.

The difference in the 2D photoexcited states density is a direct consequence of the different layer number

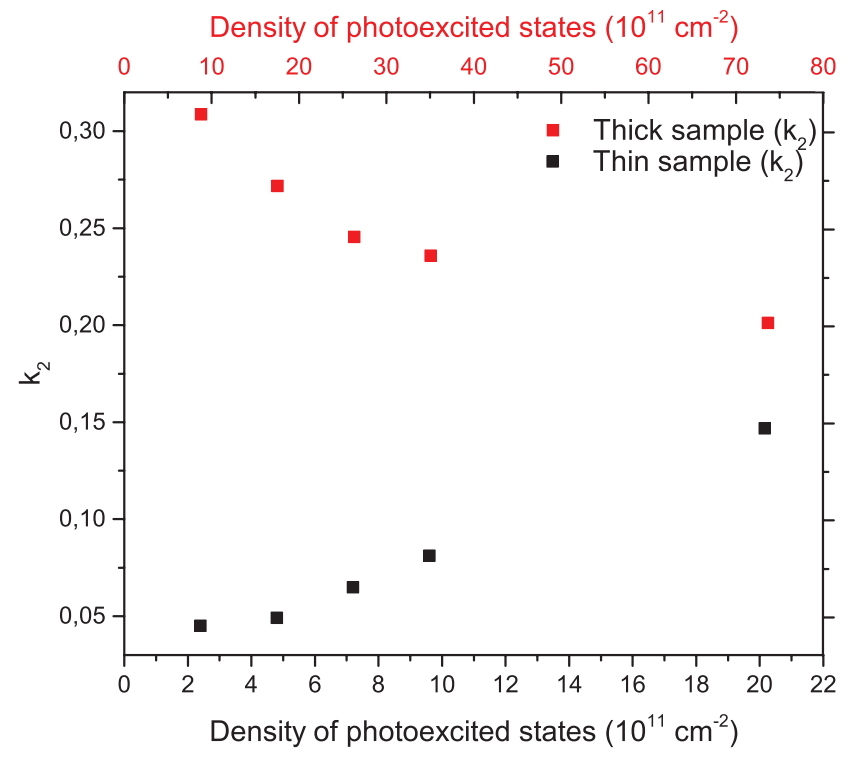

Figure $\mathbf{S} 2 \mathrm{k} 2$ of thick and thin sample as a function of the 2D excitation density

\section{S1.3.1 Linear and non linear regime}

The following figure shows a linear growth of the $\frac{\Delta T}{T}$ signal at $2.37 \mathrm{eV}$ with pump power. This indicates we are not in the saturation regime. 


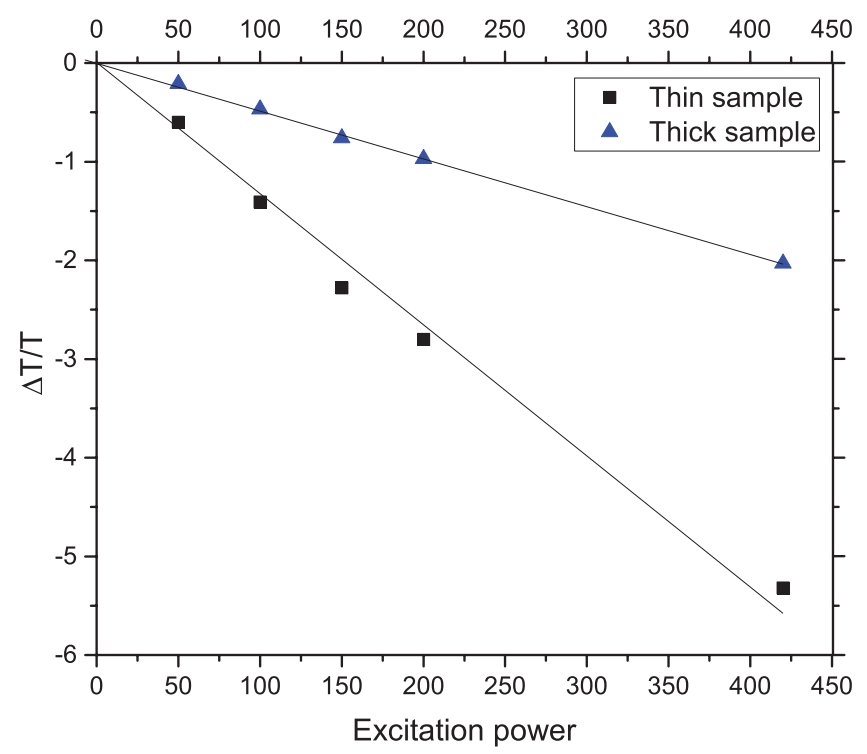

Figure S3 $\frac{\Delta T}{T}$ signal intensity power dependence at $2.38 \mathrm{eV}$.

The following plot shows the pump probe signal of the A and B exciton photobleaching as a function of the excitation power. It is clear that the A and B exciton signal is not in a linear regime.

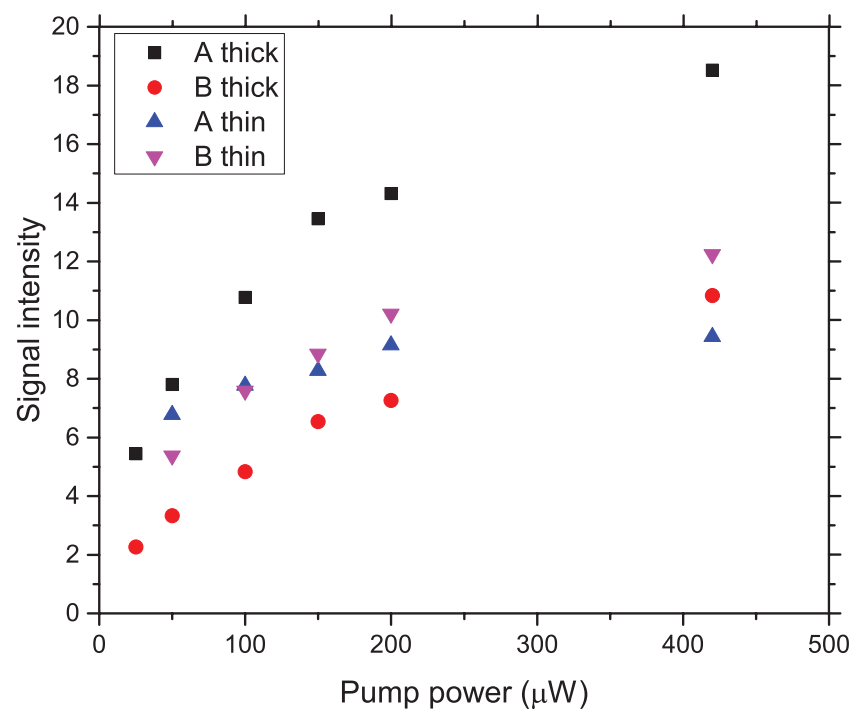

Figure S4 A and B exciton signal intensity as a function of the pump power

\section{S2 Calculating the trap density}

Following the assignment of the $k_{2}$ dynamics to charge trapping in the thick and charge recombination in the thin sample, we model the intensity dependent dynamics in order to determine the trap density. To this end, we replace Eq. 4 in the main manuscript with

$$
\frac{d C(t)}{d t}=k_{1} E(t)-\gamma C^{2}(t)-\gamma C(t) T(t)
$$

Where $\gamma$ is the rate parameter of bimolecular recombination and $T(t)$ is the time dependent density of active traps. Since recombination and trapping are both related to diffusion, we assume that they have the same rate parameter. Hence, in both samples both processes are active, but recombination dominates for $C \gg T$ and trapping for $T \ll C$. Traps are active only when they are not occupied, hence trapping of a charge deactivates one trap: 


$$
\frac{d T(t)}{d t}=-\gamma C(t) T(t)
$$

When fitting the dynamics for the five different intensities with the same parameters, we obtain $\gamma=$ $4 \times 10^{-13} \mathrm{~cm}^{2} \mathrm{~s}^{-1}$ and $T(0)=1.5 \times 10^{12} \mathrm{~cm}^{-2}$ for the thick and $\gamma=1.5 \times 10^{-13} \mathrm{~cm}^{2} \mathrm{~s}^{-1}$ and $T(0)=1.6 \times 10^{11}$ $\mathrm{cm}^{-2}$ for the thin sample. Since $\gamma$ is related to the diffusion constant, which in turn is proportional to the charge mobility via the Einstein relation, our result suggests a higher charge mobility in the thicker flake. This is in good agreement with previous studies, which showed that the highest mobility values are obtained for $\langle N\rangle=7-9$, and the mobility for $\langle N\rangle=2.5$ is $\sim 4.5$ times lower ${ }^{2-4}$. Also, the thicker sample has one order of magnitude more traps than the thinner one, as discussed in the main text.

\section{S3 $\quad k_{1}$ thickness fluence dependence.}

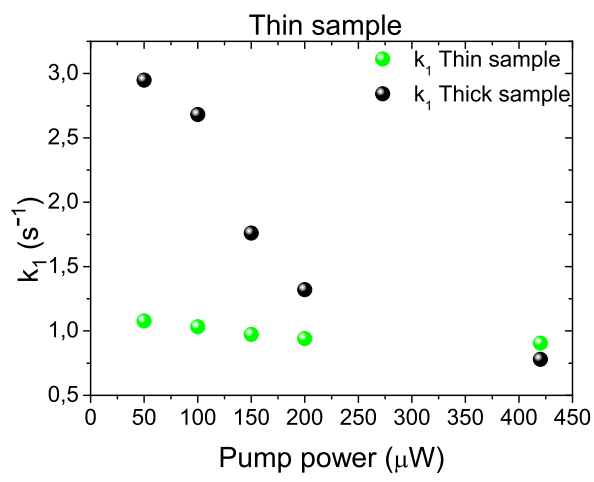

Figure S5 $k_{1}$ thickness fluence dependence.

\section{References}

[1] C. Backes, R. J. Smith, N. McEvoy, N. C. Berner, D. McCloskey, H. C. Nerl, A. O’Neill, P. J. King, T. Higgins, D. Hanlon, N. Scheuschner, J. Maultzsch, L. Houben, G. S. Duesberg, J. F. Donegan, V. Nicolosi, and J. N. Coleman, "Edge and confinement effects allow in situ measurement of size and thickness of liquid-exfoliated nanosheets," Nat. Commun., vol. 5, p. 4576, 2014.

[2] M.-W. Lin, I. I. Kravchenko, J. Fowlkes, X. Li, A. A. Puretzky, C. M. Rouleau, D. B. Geohegan, and K. Xiao, "Thickness-dependent charge transport in few-layer mos $_{2}$ field-effect transistors," Nanotechnology, vol. 27, p. 165203, 2016.

[3] S.-L. Li, K. Wakabayashi, Y. Xu, S. Nakaharai, K. Komatsu, W.-W. Li, Y.-F. Lin, A. Aparecido-Ferrera, and K. Tsukagoshi, "Thickness-dependent interfacial coulomb scattering in atomically thin field-effect transistors.," Nano. Lett, vol. 13, pp. 3546-3552, 2013.

[4] H. Schmidt, F. Giustiniano, and G. Eda, "Electronic transport properties of transition metal dichalcogenide field-effect devices: surface and interface effects," Chem. Soc. Rev., vol. 44, pp. 7715-7736, 2015. 